\title{
Singleton term breech presentation: planned vaginal delivery vs elective caesarean section at Aminu Kano Teaching Hospital
}

\author{
Omole-Ohonsi Abiodun ${ }^{1}$, Umoru Joseph ${ }^{1}$, Aiyedun Tajudeen ${ }^{2}$ \\ Sri Lanka Journal of Obstetrics and Gynaecology 2012; 34: 45-50
}

\begin{abstract}
Background: In developed countries, elective caesarean section is employed in nearly all the cases of singleton breech presentation at term, because of the safety of the procedure. However, in developing countries, where the safety and acceptance of caesarean delivery is poor, a study of the acceptable mode of delivery is needed.
\end{abstract}

Objective: To compare the pregnancy outcomes of planned vaginal delivery and elective caesarean section, among booked women with singleton term breech presentation, at Aminu Kano Teaching Hospital, Kano, Nigeria.

Methods: A 3-year cross sectional study of 135 cases of singleton term breech deliveries.

Results: The period prevalence of singleton term breech delivery among booked patients was $2.1 \%$ of the total deliveries. Elective caesarean section was used significantly more among teenagers and primigravida, while planned vaginal breech delivery was used significantly more among the women in the 25-29 and 30-34 years age group and multigravida. Maternal and neonatal morbidity did not show any significant difference between the two groups, and there was no maternal or perinatal mortality in the two groups.

Conclusion: The mode of delivery among booked patients with singleton term breech presentation at Aminu Kano Teaching Hospital should still be individualized.

Key words: singleton term breech presentation, planned vaginal delivery, elective caesarean section, fetomaternal outcome.

\footnotetext{
${ }^{1}$ Department of Obstetrics and Gynaecology, Bayero University, Aminu Kano Teaching Hospital, Kano, Nigeria.

${ }^{2}$ Federal Medical Centre, Gusau, Nigeria.

Correspondence: Omole-Ohonsi Abiodun

E-mail:aomohonsi@yahoo.com
}

\section{Introduction}

A breech birth is the birth of a baby from a breech presentation ${ }^{1}$. In breech presentation, the baby enters the birth canal with the buttocks or feet first, as opposed to the normal head first (cephalic) presentation ${ }^{1}$. Breech presentation, presents some hazards to the baby during the process of birth, with considerably increased perinatal mortality and morbidity, which makes the mode of delivery to be controversial in the field of obstetrics $^{2}$. Vaginal breech deliveries were previously the norm, until 1959, when Wright ${ }^{3}$ proposed that all breech presentations, should be delivered abdominally to reduce perinatal morbidity and mortality.

Since then, the mode of delivery of term breeches have been controversial, while some studies have reported no difference in perinatal outcomes following vaginal or abdominal delivery ${ }^{4}$, others have recommended elective caesarean section ${ }^{1,5}$. With the results of the "Term Breech Trial" 6 , a large multicentre study, that found that a elective caesarean section reduced the overall risk of perinatal death for the fetus at term in frank or complete breech presentation by $75 \%{ }^{6}$. The American College of Obstetricians and Gynecologists (ACOG) ${ }^{7}$, have recommended that all singleton fetuses at term in breech presentation, should be delivered by elective caesarean section, because it improves the outcome for breech babies, and the safety of the procedure for the mother ${ }^{1,2}$.

In developing countries like Nigeria, the situation is different. Maternal morbidity that is associated with elective caesarean section is unacceptably high, with a range of $17-25 \%$, and maternal mortality of $5-9 \% \%^{8-13}$. There is aversion to caesarean section, and one of the future implications of routine use of caesarean delivery is ruptured uterus, because the women may attempt to deliver outside the health facilities, because of fear of repeat caesarean section ${ }^{8}$. These, together with the economic implications in communities where majority of the populace live below poverty level ${ }^{8,14}$, will advise selective rather than routine use of caesarean delivery, in the management of singleton term breech presentation $^{8}$.

It is against this background that this study was designed, to compare the pregnancy outcomes following planned vaginal delivery and elective 
caesarean section, among booked patients carrying singleton term breech pregnancies at Aminu Kano Teaching Hospital, Kano, Nigeria, in order to make recommendations, which will assist policy makers in formulating acceptable and safer plan of management for women carrying singleton term breech pregnancies.

\section{Methodology}

This is a 3-year cross sectional study of all cases of singleton term breech presentation, among booked patients that were managed according to our clinical protocol at Aminu Kano Teaching Hospital, Kano, Nigeria, from January 2006 to December 2008. The women were informed of the risks and benefits of a planned vaginal breech delivery and elective caesarean section. Their choice of the mode of delivery was respected and informed written consent was obtained from the patients, or from the patients' relatives in the event of mothers that were less than 18 years. Consent discussion and chosen plan were well documented in the case notes for use by the antenatal clinic and labour room staff, and the women were provided with the best possible in-hospital care. All the vaginal deliveries were assisted breech deliveries. The deliveries, either vaginal breech delivery or caesarean section were carried out by specialist obstetricians, either consultants or senior registrars, who were assisted by other junior doctors, nursing staff, anaesthetists and paediatricians with their team of health care professionals that are skilled in neonatal resuscitation.

The women for planned vaginal breech delivery were selected and managed according to our clinical protocol for eligibility and intrapartum management. The inclusion criteria were singleton fetus in a frank or complete breech presentation at term, in the absence of a contraindication to vaginal delivery. Multiple pregnancies were exclusion criteria in this study.

The inclusion criteria for elective caesarean section were women with a contraindication to assisted vaginal delivery, which were cases with other obstetric factors like previous caesarean section, placenta praevia, intrauterine growth restriction, medical disorders like severe preeclampsia and diabetes mellitus in the antenatal period. At 36 weeks gestation, ultrasound scan and clinical pelvimetry were performed, and those with fetal growth restriction, an estimated fetal weight $>3.5 \mathrm{~kg}$, fetal attitude of extension, footling breech, and those with clinically inadequate maternal pelvis and cord presentation, were advised to have an elective caesarean section.
Intrapartum management included partographic monitoring of labour, use of adequate analgesia, electronic fetal heart monitoring in the first and second stages of labour, and psychotherapy. Following artificial or spontaneous rupture of the fetal membranes, immediate vaginal examination was performed to rule out cord prolapse. With slow progress in labour, caesarean section was advised, as it may be a warning sign of fetopelvic disproportion, and the use of oxytocin in management of breech delivery may result in premature bearing down and entrapment of the fetal head by a non-fully dilated cervix.

The first phase of the second stage of labour, which was not allowed to last more than 90 minutes, was managed passively without active bearing down effort, to allow the breech to climb the perineum. The second phase of the second stage of labour, which is the phase of active pushing, commenced after breech had climbed the perineum, and episiotomy was given except in a grand multipara with a very lax outlet. If in the first phase of the second stage of labour, the breech had not climbed the perineum after 90 minutes, or in the second phase of the second stage of labour delivery was not imminent after 60 minutes, caesarean section was recommended because of the risk of fetopelvic disproportion.

For the purpose of this study, planned vaginal breech delivery was one that was scheduled well before labour began, with the indication being either doctor's requested, which was based on medical indications, or patient-requested, which may be without medical indications. Term was between 37 and 42 completed weeks of gestation. Intrauterine growth restriction (IUGR) was diagnosed in babies, whose birth weights were below the 5th percentile of their expected weights for that period of gestation in our population. Postpartum haemorrhage (PPH) was blood loss of $500 \mathrm{ml}$ or more following vaginal delivery, and $1000 \mathrm{ml}$ or more following caesarean section. Wound sepsis was said to occur if there were local erythema, indurations and swelling of the wound edges, discharge of pus or wound dehiscence. Birth asphyxia as an APGAR score of less than 7 at 5 minutes of life. Early neonatal sepsis refers to the presence of a blood stream bacterial infection in the setting of fever, presenting in the first 7 days of life, and perinatal mortality refers to the sum of the stillbirths (deaths between the 28th week of gestation and before delivery the baby) and deaths in the first week of life.

The study variables of interest were maternal age and parity, perinatal mortality and neonatal morbidity, maternal morbidity and mortality. 
The data obtained were recorded using tables. Discrete data were presented using percentages and frequencies, continuous data were presented using mean and standard deviation. Chi-square test and Fisher exact test were used to compare discrete variables for statistical significance. A P-value of less than 0.05 was considered significant.

\section{Results}

During the study period, there were 135 cases of booked singleton term breech delivery out of a total of 6,438 deliveries, giving a period prevalence of $2.1 \%$ of the total deliveries. There was no feto-maternal morbidity or mortality.

Planned vaginal breech delivery was carried out in $61(46.2 \%)$ patients, while 71 patients $(53.8 \%)$ had elective caesarean section. There were $3(4.7 \%)$ cases of emergency caesarean section for failed planned vaginal delivery due to slow progress in labour. Indications for elective caesarean section were primgravidity 25 cases $(35.2 \%)$, previous caesarean section cases 16 cases $(22.5 \%)$, medical disorders in pregnancy 15 cases $(21.1 \%)$, footling breech presentation 8 cases $(11.3 \%)$, 'request caesareans' 5 cases $(7.0 \%)$ and big baby 2 cases $(2.8 \%)$. There was only one case of post operative wound sepsis, and 2 cases of episiotomy wound sepsis.

Table 1 describes the distribution of maternal age and parity by mode of delivery among patients with term breech delivery. The age range among the patients was 16-39 years, with a mean age of $28.4 \pm 3$.6 years. The parity range was from $0-11$, with a mean parity of 4.3 \pm 2.6 .

Table 2 describes the neonatal and maternal outcomes by mode of delivery among patients with term breech delivery. The SCBU admissions were for birth asphyxia (4 cases) and early neonatal sepsis (1 case) among the assisted vaginal delivery group while 1 case of birth asphyxia among the elective caesarean section group. There was no birth injury, perinatal or maternal mortality in the two groups.

Table 1. The distribution of maternal age and parity by mode of delivery among patients with term breech delivery

\begin{tabular}{lcccc}
\hline $\begin{array}{l}\text { Variable } \\
\text { age in years }\end{array}$ & $\begin{array}{c}\text { VBD }(\%) \\
n=61(\%)\end{array}$ & $\begin{array}{c}\text { ECS }(\%) \\
n=71(\%)\end{array}$ & Test & P-value \\
\hline$\leq 19$ & $2(3.3)$ & $21(21.1)$ & Chi-square $=14.00$ & $<0.001$ \\
$20-24$ & $8(13.1)$ & $11(19.7)$ & Chi-square $=0.02$ & 0.889 \\
$25-29$ & $26(52.5)$ & $15(22.5)$ & Chi-square $=6.11$ & 0.013 \\
$30-34$ & $19(27.9)$ & $10(28.2)$ & Chi-square $=4.62$ & 0.032 \\
$35-39$ & $6(3.3)$ & $14(8.5)$ & Chi-square $=1.78$ & 0.182 \\
Parity & & & & \\
0 & - & $25(35.2)$ & Chi-square $=24.25$ & $<0.001$ \\
$1-4$ & $38(62.3)$ & $28(39.4)$ & Chi-square $=5.97$ & 0.015 \\
$\geq 5$ & $23(37.7)$ & $18(25.4)$ & Chi-square $=1.80$ & 0.180 \\
\hline
\end{tabular}

$\mathrm{VBD}=$ vaginal breech delivery

$\mathrm{ECS}=$ elective caesarean section 
Table 2. The neonatal and maternal outcomes by mode of delivery among patients with term breech delivery

\begin{tabular}{lcccc}
\hline Variable & $\begin{array}{c}\text { VBD }(\%) \\
n=61\end{array}$ & $\begin{array}{c}\text { ECS }(\%) \\
n=71\end{array}$ & Test & P-value \\
\hline Birth asphyxia & $4(6.6)$ & $1(1.4)$ & Fisher exact & 0.181 \\
Early neonatal sepsis & $1(1.6)$ & - & Fisher exact & 0.462 \\
SCBU admission & $5(8.2)$ & $1(1.4)$ & Fisher exact & 0.095 \\
Postpartum haemorrhage & $1(1.6)$ & $2(2.8)$ & Fisher exact & 1.000 \\
Urinary tract infection & $1(1.6)$ & $3(4.2)$ & Fisher exact & 0.624 \\
Blood transfusion & $1(1.6)$ & $1(1.4)$ & Fisher exact & 1.000 \\
\hline
\end{tabular}

$\mathrm{VBD}=$ vaginal breech delivery

ECS $=$ elective caesarean section

\section{Discussion}

The period prevalence of singleton term breech delivery of $2.1 \%$ in this study is similar to $2.1-3.1 \%$ from Nigeria ${ }^{9-13}$, and 3-4\% from Europe ${ }^{2}$ and USA ${ }^{15}$, probably because the prevalence of breech presentation at term is between 2 to $4 \%$ worldwide ${ }^{16}$. Elective caesarean section rate of $53.8 \%$ among women with singleton term breech presentation in this study, is higher than $33.3 \%$ from $\mathrm{Nnewi}^{9}$, and $37.1 \%$ from Calabar ${ }^{10}$ in studies from Nigeria, probably because elective caesarean section is routinely employed, in the management of primigravida with singleton breech presentation at term in our hospital. It is however lower than $90.4 \%$ that was reported from USA ${ }^{7}$, probably because elective caesarean section is now employed, in nearly all cases of breech presentation at term, because of the safety of caesarean section, increasing 'request caesareans', and litigations that are associated with vaginal breech delivery in their obstetric practice ${ }^{15}$.

In this study, the use of elective caesarean section was significantly associated with primigravidity and teenage pregnancies, probably because majority of the teenagers were primigravidae, and in our hospital, primigravida with singleton term breech presentation are routinely offered planned caesarean section, because a primigravid labour is a trial of labour, because the pelvis had not been "tested", and there is no place for trial of labour in the conduct of vaginal breech delivery ${ }^{16,17}$. Planned vaginal breech delivery was significantly associated with multigravidity and women in the 25-29 and 30-34 years age group, probably because most of the women in the 25-29 and 30-34 years age group were multigravida, who were more suitable for assisted vaginal delivery, because their pelvis had been "tested"16,17.
There was no significant difference in the use of planned vaginal breech delivery and planned caesarean section among women in the age groups 20-24 years, probably because primigravida and multipara with singleton term breech presentation coexisted among the women in this age group, which agrees with the findings of other studies ${ }^{18}$. Also there was no significant difference in the use of planned vaginal breech delivery and elective caesarean section among grandmultipara and women in the age groups 35-39 years, probably because majority of the grandmultipara who were suitable for vaginal delivery, were in the 35-39 years age group, where older maternal age, which may be associated with medical and obstetric complications, like hypertension, diabetes, placental complications, macrosomic infants that may require a elective caesarean section existed ${ }^{8}$.

The frequency of birth asphyxia, early neonatal sepsis and SCBU admissions were significantly higher among breech fetuses at term that were delivered by vaginal route, which agrees with other studies worldwide 2,14 , probably because there is inevitable compression of the umbilical cord whiles the fetal head is in the pelvis during a vaginal breech birth ${ }^{14}$. This inevitable compression of the umbilical cord during the delivery of the fetal head, may account for why birth asphyxia is significantly associated with vaginal breech delivery, even in developed countries, where obstetric care is sophisticated. Timing is critical to an optimal outcome, and the art of obstetric judgments in the management of these patients is important, in order to avoid delay in the delivery of the fetal head ${ }^{4}$.

Early neonatal sepsis occurred more among the babies that were delivered by assisted breech delivery, 
probably because of acquired infection from the birth canal during the passage of the baby ${ }^{8}$. This together with higher occurrence of birth asphyxia, may explain why admission into SCBU, was significantly more among the babies that were delivered by planned assisted breech delivery protocol, but there was no perinatal death in the two groups, probably because of good protocol of selection of the mode of delivery among the women, and meticulous care, as the deliveries were conducted by senior registrars or consultants, with efficient backup from anaesthetic, neonatologists and other relevant medical staff who are experienced with breech births. This agrees with the findings of similar studies ${ }^{4}$, probably because the skill of the birth attendants, especially experience with breech births is of crucial importance, as many of the dangers in vaginal birth for breech babies come from mistakes made by birth attendants ${ }^{4}$.

However, it did not agree with the findings of Conde-Agudelo et al in Latin America ${ }^{14}$, where breech fetuses that were delivered by vaginal route had almost nine times increased risk of perinatal mortality, probably because of inadequate manpower and facilities, as some of their cases were not managed by specialist obstetricians. This will require community campaign for effective and active antenatal care and hospital delivery in developing countries, where majority of the patients are unbooked, as well as capacity building, which should include training and re-training of birth attendants in the art of vaginal breech delivery.

In this study, maternal morbidity occurred with higher frequency among the women who had elective caesarean delivery, but there was no significant difference in the occurrence of maternal morbidity between the two groups, which agrees with other studies worldwide ${ }^{1,2,5,6,10}$, probably because elective caesarean section, like any major surgery, involves risks ${ }^{6}$. Meticulous care and involvement of skilled birth attendants in the form of senior obstetricians, may explain why there was no significant difference in the occurrence of maternal morbidity between the two groups in this study, which agrees with studies from developed countries like USA ${ }^{6}$ and Europe ${ }^{2}$. This may also explain why there was no maternal mortality in both groups in this study.

However, this does not agree with the findings of some studies from developing countries, where the maternal morbidity and mortality that is associated with elective caesarean section is unacceptably high, which has been attributed to inadequacy of skilled manpower and facilities to conduct caesarean section $^{14}$. Capacity building, which should include provision of facilities, as well as training and re- training of medical personnel including the doctors, in the conduct of vaginal breech delivery and caesarean section should be encouraged.

There was no feto-maternal morbidity or mortality, among the cases that had emergency caesarean section for failed planned vaginal breech delivery in this study, probably because of involvement of skilled birth attendants, partographic monitoring of labour and use of continuous electronic fetal monitoring in labour, which may have timely decided when to terminate the labour in favour of emergency caesarean section, before deterioration in feto-maternal condition ensued. Furthermore, facilities for caesarean section, neonatal care and blood products for transfusion were available 24 hours of the day. This further emphasized the need for capacity building in developing countries, in order to ensure provision of adequate facilities and skilled birth attendants, with pregnancy outcomes that are similar to that of developed countries.

This study showed that with well designed protocols, proper selection of the mode of delivery, and management of labour/delivery by skilled birth attendants, with backup from relevant medical staff who are experienced in breech births, and availability of facilities for caesarean section, neonatal care and blood transfusion 24 hours of the day, the pregnancy outcomes with planned vaginal delivery and elective caesarean section, among booked women with singleton term breech presentation, did not show statistically significant difference. In developing countries, provided adequate health care is available, the mode of delivery should be individualized. Routine use of elective caesarean section for singleton term breech presentation, may not be applicable, because there is aversion to caesarean section, desire for a large family size, women who cannot achieve vaginal delivery are perceived as reproductive failures, and the resource implications of caesarean delivery ${ }^{8,18}$, may make women with previous caesarean section scars, to be at risk of ruptured uterus in subsequent pregnancies, because they may prefer to labour/ deliver outside the health facilities, for fear of repeat caesarean section.

The use of external cephalic version at term should be re-visited in our obstetric practice in developing countries, where there is high prevalence of cephalopelvic disproportion ${ }^{8}$, so that primigravida, as is the case in Aminu Kano Teaching Hospital, and women with mild to moderate cephalopelvic disproportion, carrying singleton term fetuses in breech presentation, can be allowed to have trial of labour following successful version, and reduce the caesarean section rate ${ }^{19}$. Because of the small sample 
size and the possible bias in this hospital based study, large multicentre studies will be required, to provide firm reassurance of safety with vaginal breech delivery in this study

\section{Conclusion and recommendations}

The mode of delivery among booked patients with singleton term breech presentation at Aminu Kano Teaching Hospital should still be individualized. Patients with singleton breech presentation at term in peripheral health facilities should be transferred to specialized health facilities, where skilled manpower and facilities for vaginal breech delivery/caesarean section are available. Health facilities that manage women with singleton term breech presentation should have their protocol, for selection of the mode of delivery and the conduct of vaginal breech delivery/ caesarean section, which should involve senior obstetricians. Capacity building, which should include provision of adequate facilities, and training and re-training of medical personnel including the doctors, in the conduct of vaginal breech delivery and caesarean section should be encouraged, especially in developing countries.

\section{References}

1. Hofmeyr GJ, Hannah ME. Planned caesarean section for term breech delivery (Cochrane Review). In: Cochrane Database of Systematic Reviews 2001; 3. CD000166.

2. Herbest A. Term breech delivery in Sweden: mortality relative to fetal presentation and planned mode of delivery. Acta Obstet Gynecol Scand 2005; 84: 593-601.

3. Wright RC. Reduction of perinatal mortality and morbidity in breech delivery, through positive use of caesarean section. Obstet Gynaecol 1959; 4: 758.

4. Kumari AS, Grundsell H. Mode of delivery for breech presentation in grandmultiparous women. Int J Gynaecol Obstet 2004; 85: 234-9.

5. Golfier F, Vaudoyer F, Ecochard R, Champion R, Audra P, Raudrant $D$. Planned vaginal delivery versus elective cesarean section in singleton term breech presentation: a study of 1116 cases. Eur J Obstet Gynecol Reprod Biol 2001; 98(2): 186-92.

6. Hannah ME, Hannah WJ, Hewson SA, Hodnett ED, Saigal S, Willan AR. Planned caeserean section versus planned vaginal birth for breech presentation at term: a randomized multicentre trial. Term Breech Trial Collaborative Group. Lancet 2000; 356: 1375-83.

7. American College of Obstetricians and Gynecologists. Mode of term singleton breech delivery. Int J Gynecol Obstet 2001; 77: 65-66.

8. Omole-Ohonsi A, Attah RA. Outcome of caesarean delivery at Aminu Kano Teaching Hospital. Kanem Journal of Medical Sciences 2009; 3(2): 36-39.

9. Abasiattai AM, Bassey EA, Etuk SJ, Udoma EJ, Ekanem AD. Caesarean section in the management of singleton breech delivery in Calabar, Nigeria. Niger J Clin Pract 2006; 9(1): 22-25.

10. Igwegbe AO, Monago EN, Ugboaja JO. Caesarean versus vaginal delivery for term breech presentation: a comparative analysis. Afr J Biomed Res 2010; 13: 15-18.

11. Fasubaa OB, Orji EO, Ogunlola O, Kuti O, Shittu SA. Outcome of singleton breech delivery in Wesley Guild Hospital, Ilesa, Nigeria. Trop J Obstet Gynaecol 2003; 20: 59-62.

12. Fawole AO, Adeyemi AS, Adewole IF, Omigbodun AO. A ten-year review of breech deliveries at Ibadan. Afr J Med Med Sci 2001; 30(1-2): 87-90.

13. Orji EO, Ajenifuja KO. Planned vaginal delivery versus caesarean section for breech presentation in Ile-Ife, Nigeria. East Afr Med J 2003; 80(11): 589-91.

14. Conde-Agudelo A, Belizan JM, Diaz-Rossello JL. Epidemiology of fetal death in Latin America. Acta Obstetricia Et Gynecologica Scandinavica 2000; 79: 371-8.

15. Carbillon L. Vaginal versus cesarean delivery for breech presentation in California: a population-based study. Obstet Gynecol 2004; 103: 1003-4.

16. Orhue AAE. Breech presentation. In: Textbook of Obstetrics and Gynaecology for Medical Students. Agboola A (eds). Heinemann Educational Books (Nigeria) Plc. Ibadan. 2006; pp. 423-9.

17. Ikpeze OC. Preterm labour and delivery. In: Contemporary Obstetrics and Gynaecology for Developing Countries. Okonofua $\mathrm{F}$ and Odunsi K (eds). First edition. Women Health and Action Research Centre, Benin City, 2003; pp. 454-61.

18. Emembolu JO, Adega AAO. A prospective study of singleton breech delivery in Zaria, Northern Nigeria. Trop J Obstet Gynaecol 1996; 13(1): 29-33.

19. Hofmeyr GJ, Kulier R. External cephalic version for breech presentation at term (Cochrane Review). In: Cochrane Database of Systematic Reviews 2001; 2. CD000083. 